\section{Birefringence of Polymers in the Softening Zone}

\section{P. H. Mott and C. M. Roland*}

Chemistry Division, Code 6120, Naval Research Laboratory, Washington, D.C. 20375-5342

Received February 5, 1998

Revised Manuscript Received J uly 31, 1998

\section{Introduction}

It is well-known that rubbers and glasses usually exhibit proportionality between the true stress, $\sigma$, and the optical birefringence, $\Delta \mathrm{n}:^{1}$

$$
\Delta \mathrm{n}=\mathrm{C} \sigma
$$

where C is the stress optical coefficient. However, in the softening zone of the viscoelastic spectrum, deviations are observed from the stress optical law, with all quantities now complex and having different frequency dependencies:

$$
\Delta \mathrm{n}^{*}(\omega)=\mathrm{C}^{*}(\omega) \sigma^{*}(\omega)
$$

where $\omega$ is the angular frequency. For analysis of data obtained in typical dynamic experiments, it is useful to divide eq 2 by the strain, $\epsilon$, to yield

$$
\mathrm{O}^{*}(\omega)=\mathrm{C}^{*}(\omega) \mathrm{E}^{*}(\omega)
$$

where $E^{*}(\omega)$ is the dynamic modulus and the strainoptical coefficient is defined as $\mathrm{O}^{*}(\omega)=\Delta \mathrm{n}^{*}(\omega) / \epsilon$.

In analyzing birefringence data in the softening zone, Read $^{2}$ assumed that the measured stress was just the sum of independent contributions from glassy and rubbery components:

$$
\sigma^{*}(\omega)=\sigma_{\mathrm{G}}^{*}(\omega)+\sigma_{\mathrm{R}}^{*}(\omega)
$$

and

$$
\mathrm{E}^{*}(\omega)=\mathrm{E}_{\mathrm{G}}^{*}(\omega)+\mathrm{E}_{\mathrm{R}}^{*}(\omega)
$$

The birefringence is then given by ${ }^{2}$

$$
\Delta \mathrm{n}^{*}(\omega)=\mathrm{C}_{\mathrm{G}} \sigma_{\mathrm{G}}^{*}(\omega)+\mathrm{C}_{\mathrm{R}} \sigma_{\mathrm{R}}^{*}(\omega)
$$

where $C_{G}$ and $C_{R}$ are the respective stress optical coefficients for the putative glassy and rubbery components. The complex strain optical coefficient can then be expressed as

$$
\mathrm{O} *(\omega)=\mathrm{C}_{\mathrm{G}} \mathrm{E}_{\mathrm{G}}^{*}(\omega)+\mathrm{C}_{\mathrm{R}} \mathrm{E}_{\mathrm{R}}^{*}(\omega)
$$

From behavior in the terminal zone, Read identified the rubbery stress optical coefficient in terms of the storage and Ioss functions: ${ }^{2}$

$$
C_{R} \equiv \lim _{\omega \rightarrow 0} \frac{O^{\prime}(\omega)}{E^{\prime}(\omega)}=\lim _{\omega \rightarrow 0} \frac{O^{\prime \prime}(\omega)}{E^{\prime \prime}(\omega)}
$$

while the glassy coefficient was assumed to be related only to the in-phase components:

$$
C_{G} \equiv \lim _{\omega \rightarrow \infty} \frac{O^{\prime}(\omega)}{E^{\prime}(\omega)}
$$

Equations 5-9 were applied to dynamic bi refringence data from four different polymers, yiel ding the frequencydependent contributions of the two components. ${ }^{2}$ Read actually referred to the glassy component as the distortional mode, involving small torsional motions of the chain. This mode can be identified with the local segmental dynamics. ${ }^{3}$ The rubbery component comprises orientational motions associated with longer length scales (i.e., the chain modes described by the Rouse model).

In a variation on this approach, Osaki and co-workers adopted the definition ${ }^{4,5}$

$$
C_{G} \equiv \lim _{\omega \rightarrow \infty} \frac{O^{\prime \prime}(\omega)}{E^{\prime \prime}(\omega)}
$$

based on the fact that in the glassy zone $\mathrm{O}^{*}(\omega) / \mathrm{E}^{*}(\omega) \approx$ $\mathrm{O}^{\prime \prime}(\omega) / \mathrm{E}^{\prime \prime}(\omega) \gg \mathrm{O}^{\prime}(\omega) / \mathrm{E}^{\prime}(\omega)$. E quations 5-8 and 10 have been applied to the interpretation of viscoelastic and birefringence data from more than a dozen polymers. ${ }^{6}$ More recently, to account for results from polyisobutylene and poly(2-vinylnaphthalene), the method was extended to include a third component for the stress: ${ }^{7,8}$

$$
E^{*}(\omega)=E_{R}^{*}(\omega)+E_{G}^{*}(\omega)+E_{F}^{*}(\omega)
$$

The last term, ostensibly accounting for "fluctuations" in the local stress, was necessary to obtain agreement with the modulus determined experimentally at high frequencies for these polymers. However, this stress does not contribute to the dynamic birefringence (i.e., $C_{F}=0$ ), whereby eq 6 is still employed for the strain optical coefficient. It is worth noting that polyisobutylene's viscoelastic behavior is, in general, somewhat unique .,10 $^{9,10}$

It is important to recognize that the approach of Read and Osaki is not corroborated by successful application of eqs 5-11 to experimental data. The only quantities not experimentally determined are the (hypothetical) components of the modulus, which from eqs 5 and 7 are given by

$$
E_{G}^{*}(\omega)=\frac{O^{*}(\omega)-C_{R} E^{*}(\omega)}{C_{G}-C_{R}}
$$

and

$$
E_{R}^{*}(\omega)=\frac{O *(\omega)-C_{G} E^{*}(\omega)}{C_{R}-C_{G}}
$$

In fact, experimental results can be described through the use of either eqs 9 or 10, albeit with different values obtained for the glassy and rubbery contributions to the total stress. We examine implications of such an analysis in the next section.

\section{Results and Analysis}

Polyisoprene. Displayed in Figure 1 are the dynamic modulus and birefringence master curves for polyisoprene (PI). ${ }^{11}$ Analysis of these data using eqs 


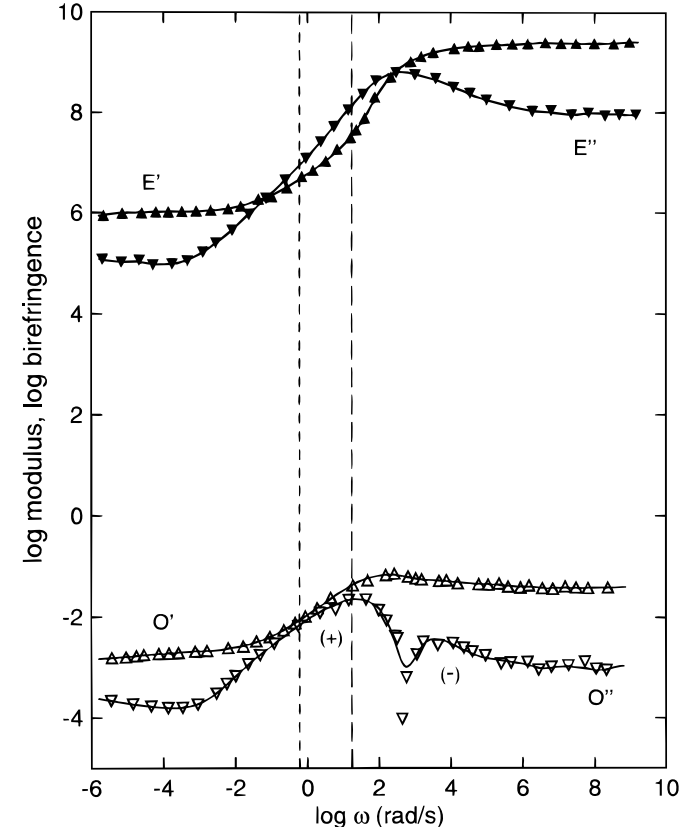

Figure 1. Experimental time-temperature master curves reported by Osaki et al. ${ }^{11}$ for the modulus (solid symbols) and the strain optical coefficient (hollow symbols) of pol yi soprene. Note the change in sign of $O^{\prime \prime}(\omega)$ at $\log \omega=2.5$. The solid lines through the data were calculated using eqs 18-20. The larger vertical dashes indicate the frequency at which the compliance attains a value 4 times larger than the high-frequency limiting compliance $\left(\approx 0.3 \mathrm{GPa}^{-1}\right)$. The smaller vertical dashes represent the position in the spectrum at which the contribution of local segmental motion to the mechanical response becomes negligible, as inferred from dielectric spectroscopy.

5-8 and 10 gives $C_{G}=-0.011$ and $C_{R}=2.0 \mathrm{GPa}^{-1}$, as reported by Osaki et al. ${ }^{11}$ From these results, eqs 12 and 13 yield the frequency-dependent contributions of the glassy and rubbery components to the modulus, whose ratio is denoted by a solid line in Figure 2 . The rubber predominates at the lowest frequencies, while the glass governs the behavior at high frequencies. More interesting is the relative contributions in the softening zone (ca. $-1<\log \omega(\mathrm{rad} / \mathrm{s})<3)$. Figure 2 implies that the glassy component makes a significant contribution to the response, indeed the dominant one, virtually throughout this region of the spectrum. The question is whether such a result is congruent with other experimental facts. For example, the loss component of the dynamic birefringence in Figure 1 changes sign at a frequency of about $50 \mathrm{~Hz}$. One might anticipate that such a sign reversal is occasioned by some change in the deformation mechanism. However, according to Figure 2 the glassy component continues to dominate the response through frequencies associated with the change in sign of $\mathrm{O}^{\prime \prime}(\omega)$.

From the creep recovery of low-molecular-weight polymers near $T_{g}$, Plazek et al. . $^{3,12-14}$ determined that the contribution from local segmental motion has essentially vanished by the time the compliance reaches a level ca. 4 times its glassy value $\left(D_{\mathrm{G}} \approx 0.3 \mathrm{GPa}^{-1}\right)$. Using the data in Figure 1 , we determine that the storage compliance $\left(D^{\prime}=E^{\prime} /\left(E^{\prime 2}+E^{\prime \prime 2}\right)\right.$ increases by this factor of 4 at $\log \omega=1.26$ (indicated by the large-dashed vertical line in Figures 1 and 2). Contrarily, the analysis of Osaki et al. ${ }^{11}$ suggests the behavior at this frequency is still governed primarily by the glassy component (Figure 2).

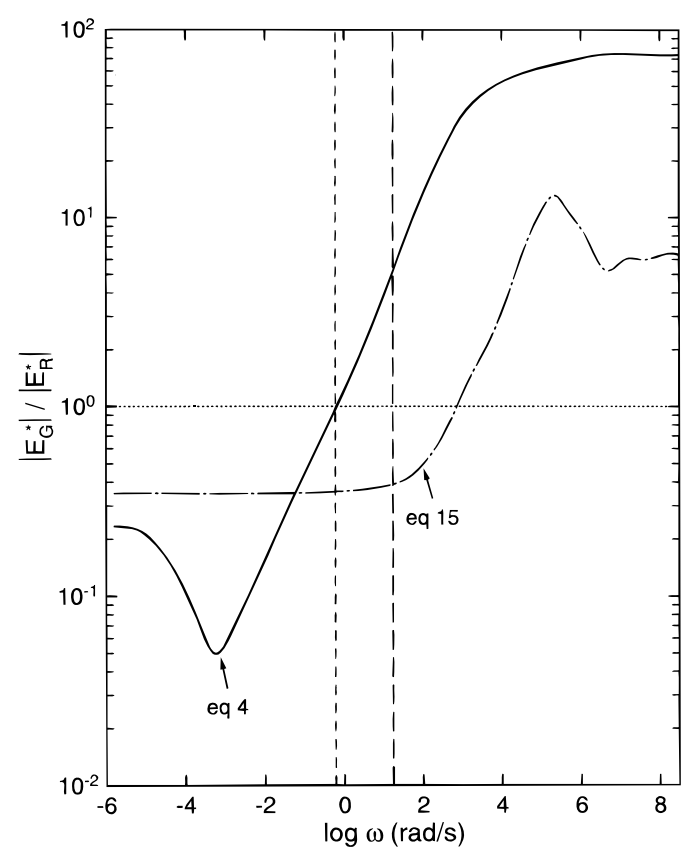

Figure 2. The relative contributions from the glassy and rubbery components to the data in Figure 1, as reflected by the ratio of the magnitudes of their respective complex moduli. The solid line was calculated assuming additivity of the stresses with $C_{G}=-0.011$ and $C_{R}=2.0$, while the dotteddashed line assumes additivity of the strains with $\mathrm{C}_{\mathrm{G}}=-0.06$ and $C_{R}=0.02$ (units of $\mathrm{GPa}^{-1}$ ). The oscillations in the former (latter) at lower (higher) frequency reflect noise in the $\mathrm{E}^{*}$ and $\mathrm{O}^{*}$ data. The vertical lines are as defined in Figure 1.

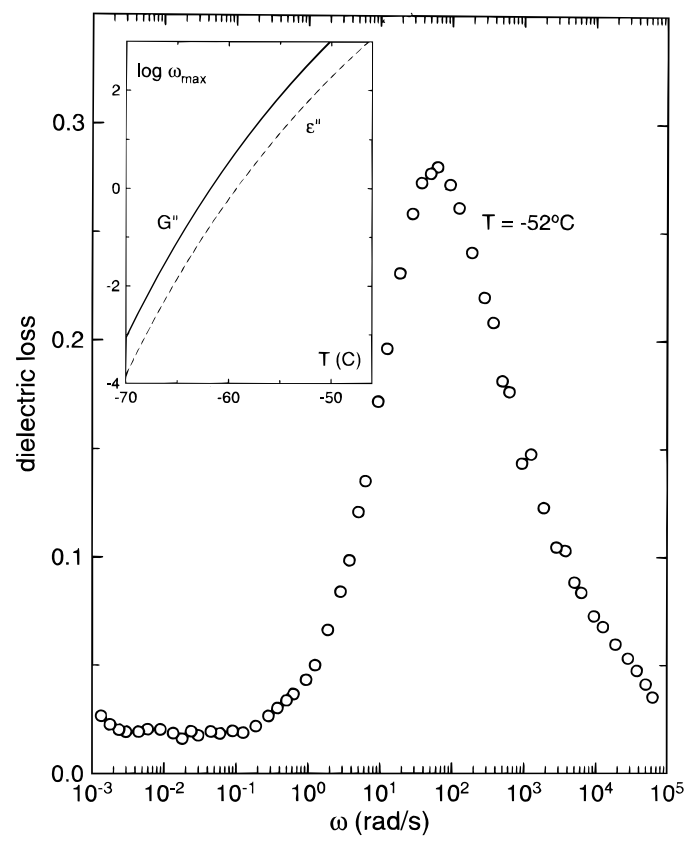

Figure 3. The dielectric loss of high-molecular-weight $\mathrm{PI}$ measured ${ }^{15}$ at the reference temperature of Figure 1 . The inset compares the frequency of the maximum in the dielectric loss (dashed line) to the maximum in the loss modulus (solid line).

The dielectric spectrum of a high-molecular-weight $\left(=5.0 \times 10^{6}\right) \mathrm{PI}$ was recently measured ${ }^{15}$ at the same temperature $\left(-52{ }^{\circ} \mathrm{C}\right)$ as the reference temperature of Figure 1. In Figure 3 we show the dispersion in the dielectric loss due to local segmental motion. Since PI has a dipole moment parallel to the chain, motion of the chain end-to-end vector gives rise to a "normal" mode, analogous to the terminal mechanical relax- 
ation. ${ }^{16,17}$ However, the molecular weight of the $\mathrm{PI}$ is sufficiently high that the normal mode peak, located at $\omega \approx 10^{-7} \mathrm{rad} / \mathrm{s},{ }^{15}$ does not overlap with the segmental peak. This resolution of the modes means that the segmental dispersion decays al most to zero at about 2.8 decades toward lower frequency from the peak maximum. This identifies the frequency, $\omega=0.1 \mathrm{rad} / \mathrm{s}$, in Figure 3 at which the contribution from the local segmental dynamics becomes negligible.

As seen in the inset to Figure 3 , the segmental relaxation peak measured mechanically occurs at a frequency almost 6 times faster than the dielectric dispersion. From the decay of the isolated dielectric dispersion (Figure 3), we can estimate the frequency at which the local segmental modes no longer contribute to the mechanical spectrum. This frequency, $\omega=0.6$ $\mathrm{rad} / \mathrm{s}$, is denoted by a short-dashed vertical line in Figures 1 and 2. However, as shown in Figure 2, the analysis of Osaki et al.11 implies a substantial glassy contribution, comparable in magnitude to the rubbery component, persisting at this low frequency. Thus, the results of Osaki et al. concerning the dynamics of the glassy mode appears to be at odds with two independent assessments of the time-scale for local segmental relaxation. In the next section we investigate an obvious cause of this discrepancy.

Strain Additivity. The method of Osaki and coworkers is based on the idea that scalar stresses (moduli) are additive (eqs 4-6). The virial stress formulation, whereby vector forces are summed to yield the system stress, is often used in molecular dynamic simulations of polymers, 18 and it has been taken as justification for eq 5.7,8 However, simulations can be analyzed using alternative conservation laws, for example, summation of the local strains. ${ }^{19-21}$

Creep/recovery experiments on a polymer melt enable the assumption underlying eq 4 to be assessed directly. The linear creep compliance, $\mathrm{D}(\mathrm{t})$, is given by the sum of the recoverable compliance, $D_{\text {rec }}(t)$ and the viscous deformation $22-24$

$$
\mathrm{D}(\mathrm{t})=\mathrm{D}_{\mathrm{rec}}(\mathrm{t})+\mathrm{t} / \eta
$$

where t and $\eta$ are time and viscosity, respectively. This means that recoverable and viscous stresses are not independent, but are governed by the corresponding elastic and viscous strains. The validity of eq 14 is confirmed from measurement of the recovery after constant stress creep. ${ }^{25}$ The remaining (permanent) deformation of a polymeric melt equals $t / \eta$ times the stress. Thus, creep/recovery experiments indicate that, at least for terminal flow, deformation processes are additive in strain, rather than in stress. We now modify the analysis of Read $^{2}$ and Osaki ${ }^{4,5}$ in consideration of this idea.

The corollary to eq 4 for the assumption that the total strain is the sum of independent glassy and rubbery components is

$$
\epsilon=\epsilon_{\mathrm{G}}+\epsilon_{\mathrm{R}}
$$

Brewster's law can be expressed as

$$
\frac{\epsilon}{\Delta \mathrm{n}^{*}}=\frac{\epsilon_{\mathrm{G}}}{\mathrm{C}_{\mathrm{G}} \sigma^{*}}+\frac{\epsilon_{\mathrm{R}}}{\mathrm{C}_{\mathrm{R}} \sigma^{*}}
$$

From eq 15 , the total compliance $D^{*}(\omega),\left(=1 / E^{*}(\omega)\right)$, is given by

$$
D^{*}(\omega)=D_{\mathrm{G}}^{*}(\omega)+D_{\mathrm{R}}^{*}(\omega)
$$

and Brewster's law becomes

$$
\mathrm{O} *(\omega)^{-1}=\frac{\mathrm{D}_{\mathrm{G}}^{*}(\omega)}{\mathrm{C}_{\mathrm{G}}}+\frac{\mathrm{D}_{\mathrm{R}}^{*}(\omega)}{\mathrm{C}_{\mathrm{R}}}
$$

The assumption of strain additivity then yields for the respective glassy and rubbery components

$$
D_{G}^{*}(\omega)=\frac{C_{R} / O *(\omega)-D^{*}(\omega)}{C_{R} / C_{G}-1}
$$

and

$$
D_{R}^{*}(\omega)=\frac{C_{G} / O *(\omega)-D^{*}(\omega)}{C_{G} / C_{R}-1}
$$

Using eqs 18-20, the data in Figure 1 can be reproduced. However, as pointed out above in reference to the analyses of Osaki and co-workers, $4,5,11$ this is a given, and not a test of the method. The components of the stress optical coefficient, $C_{G}$ and $C_{R}$, are no longer defined by eqs 8-10; in fact, they have no a priori values, other than the requirement of yielding agreement with the experimental data of Figure 1.

To demonstrate this, the master curves in Figure 1 were fitted using $\mathrm{C}_{\mathrm{G}}=-0.06$ and $\mathrm{C}_{\mathrm{R}}=0.02 \mathrm{GPa}^{-1}$ (shown by the solid lines through the data points). Using eqs 19 and 20 we then obtain the relative contributions of the glassy and rubbery components, which are denoted by the dotted-dashed line in Figure 2. The glassy contribution is now found to be significantly less than that from the rubber through most of the transition zone. In fact, the strain optical coefficient, $\mathrm{O}^{\prime \prime}(\omega)$, changes sign at a frequency in Figure 1 at which the rubber begins to replace the glassy component as the dominant contributor to the overall response.

We also note that for both measures of the position in the spectrum at which the glassy component's contribution becomes negl igible- $\mathrm{D}(\mathrm{t})$ attaining a value 4 times higher than the glassy compliance ${ }^{3,12-14}$ (largedashed line in Figure 2) and decay of the local segmental relaxation function to zero ${ }^{15}$ (short-dashed line in Figure 2)-eqs 15-20 indicate dominance of the rubbery component. Thus, the assumption of strain additivity enables the experimental data to be accurately reproduced and the deduced contributions of the glassy and rubber components are consistent with alternative methods of assessing their relative significance.

\section{Summary}

The interplay of forces in a dynamically correlated system such as polymer melts may be too complicated for any simpleanalysis to be correct. Nevertheless, such efforts can potentially yield useful insights. The work described herein is not meant to suggest that an interpretation based on the summation of strains is necessarily valid. However, this approach leads to results consistent with four experimental facts:

(i) The el astic and viscous deformations in the terminal zone are additive in the strain. ${ }^{22-25}$

(ii) There is a change in sign of the imaginary part of the strain optical coefficient in the high-frequency end of the transition zone. ${ }^{11}$ 
(iii) As determined by creep experiments, the decay of the glassy compliance occurs toward higher frequencies in the transition zone. 3,12-14

(iv) As determined by dielectric spectroscopy, the local segmental relaxation decays to zero near the middle of the transition zone. ${ }^{15}$

These results should prompt reexamination of the assumption of stress additivity and any inferences derived from it.

Acknowledgment. Insightful discussions with K.L. Ngai and D.J . Plazek are gratefully acknowledged. This work was supported by the Office of Naval Research.

\section{References and Notes}

(1) Riande, E.; Saiz, E. Dipole Moments and Birefringence of Polymers; Prentice Hall: Englewood Cliffs, NJ , 1992.

(2) Read, B. E. Polym. Eng. Sci. 1983, 23, 835.

(3) Ngai, K. L.; Plazek, D. J. Rubber Chem. Technol. 1995, 68 376.

(4) Inoue, T.; Okamoto, H.; Osaki, K. Macromol ecules 1991, 24, 5670.

(5) Inoue, T.; Hayashihara, H.; Okamoto, H.; Osaki, K. J . Polym. Sci., Polym. Phys. Ed. 1992, 30, 409.

(6) Osaki, K.; Inoue, T.; Hwang, E.-J .; Okamoto, H.; Takiguchi, O. J. Non-Cryst. Solids 1994, 172-174, 838.

(7) Osaki, K.; Okamoto, H.; Inoue, T.; Hwang, E.-J . Macromolecules 1995, 28, 3625 .

(8) Okamoto, H.; Inoue, T.; Osaki, K. J . Polym. Sci., Polym. Phys. Ed. 1995, 33, 1409.
(9) Plazek, D. J .; Chay, I.-C.; Ngai, K. L.; Roland, C. M Macromolecules 1995, 28, 6432.

(10) Santangelo, P. G.; Ngai, K. L.; Roland, C. M. Macromolecules 1993, 26, 2682.

(11) Okamoto, H.; Inoue, T.; Osaki, K. J . Polym. Sci., Polym. Phys. 1985, 33, 417.

(12) Plazek; D. J .; O'Rourke, V. M. J . Polym. Sci. A-2 1971, 9, 209.

(13) Ngai, K. L.; Plazek, D. J .; Echeverria, I. Macromolecules 1996, 29, 7937.

(14) Ngai, K. L.; Plazek, D. J .; O'Rourke, V. M. Macromol ecules 1997, 30, 5450.

(15) Santangelo, P. G.; Roland, C. M. Macromolecules 1998, 31 3715.

(16) Adachi, K.; Kotaka, T. Prog. Polym. Sci. 1993, 18, 585.

(17) Adachi, K.; Kotaka, T. Macromolecules 1985, 18, 466.

(18) See, for example, Gao, 」 : Weiner, J. H. Macromolecules 1987, 20, 2520. Gao, J.; Weiner, J. H. Science 1994, 266, 748.

(19) Argon, A. S.; Kuo, H. Y. Mater. Sci. Eng. 1979, 39, 101.

(20) Deng, D.; Argon, A. S.; Yip, S. Philos. Trans. R. Soc. London, Ser. A 1989, 329, 613.

(21) Mott, P. H.; Argon, A. S.; Suter, U. W. Philos. Mag. A 1993, 67, 931.

(22) Ferry, J . D. Viscoel astic Properties of Polymers; Wiley: New York, 1980; Chapter 1.

(23) Leaderman, H. Rheology 1958, 2, 1.

(24) Bird, R. B.; Armstrong, R. C.; Hassager, O. Dynamics of Polymeric Liquids; Wiley: New York, 1977; Vol. I, p 164

(25) Plazek, D. J . J . Rheol. 1992, 36, 1671. MA980174P 